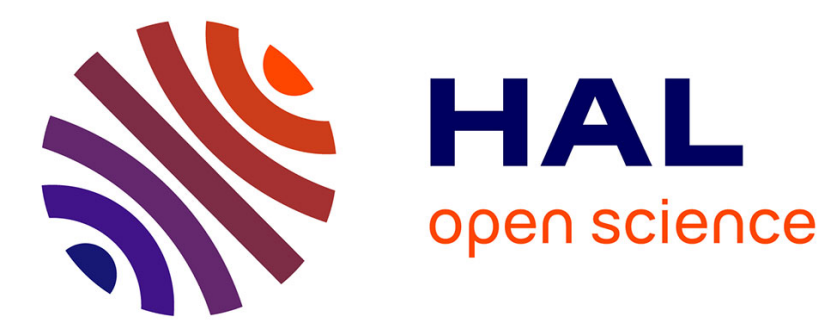

\title{
Mechanism of plasmon-mediated enhancement of photovoltaic efficiency
}

\author{
W Jacak, J Krasnyj, J Jacak, W Donderowicz, L Jacak
}

\section{To cite this version:}

W Jacak, J Krasnyj, J Jacak, W Donderowicz, L Jacak. Mechanism of plasmon-mediated enhancement of photovoltaic efficiency. Journal of Physics D: Applied Physics, 2011, 44 (5), pp.55301. 10.1088/0022-3727/44/5/055301 . hal-00591293

\section{HAL Id: hal-00591293 \\ https://hal.science/hal-00591293}

Submitted on 9 May 2011

HAL is a multi-disciplinary open access archive for the deposit and dissemination of scientific research documents, whether they are published or not. The documents may come from teaching and research institutions in France or abroad, or from public or private research centers.
L'archive ouverte pluridisciplinaire HAL, est destinée au dépôt et à la diffusion de documents scientifiques de niveau recherche, publiés ou non, émanant des établissements d'enseignement et de recherche français ou étrangers, des laboratoires publics ou privés. 


\title{
Mechanism of plasmon-mediated enhancement of PV efficiency
}

\author{
W. Jacak \\ Institute of Physics, Wrocław University of Technology, Wyb. Wyspiańskiego 27, \\ 50-370 Wrocław, Poland \\ E-mail: witold.aleksander.jacak@pwr.wroc.pl

\section{J. Krasnyj} \\ Group of Theor. Physics, International University, Fontanskaya Doroga 33, 65-026 \\ Odessa, Ukraine
}

\section{J. Jacak}

Institute of Physics, Wrocław University of Technology, Wyb. Wyspiańskiego 27, 50-370 Wrocław, Poland

\section{W. Donderowicz}

Institute of Physics, Wrocław University of Technology, Wyb. Wyspiańskiego 27, 50-370 Wrocław, Poland

\section{Jacak}

Institute of Physics, Wrocław University of Technology, Wyb. Wyspiańskiego 27, 50-370 Wrocław, Poland

\begin{abstract}
.
Metallic nanospheres $(\mathrm{Au}, \mathrm{Ag}, \mathrm{Cu})$ deposited on $\mathrm{PV}$-active semiconductor surface can act as light converters, collecting energy of incident photons in plasmon oscillations. This energy can be next transferred to semiconductor substrate via a near-field channel, in a more efficient manner in comparison to the direct photo-effect. We explain this enhancement by inclusion of indirect inter-band transitions in semiconductor layer due to the near-field coupling with plasmon radiation in nanoscale of the metallic components, where the momentum is not conserved as the system is not translationally invariant. The model of the nano-sphere plasmons is developed (RPA, analytical version, adjusted to description of large metallic clusters, with radius of $10-60 \mathrm{~nm}$ ) including surface and volume modes. Damping of plasmons is analyzed via Lorentz friction, and irradiation losses in far- and near-field regimes. Resulting resonance shifts are verified experimentally for $\mathrm{Au}$ and $\mathrm{Ag}$ colloidal water solutions with respect to particle size. Probability of the electron interband transition (within the Fermi golden rule) in substrate semiconductor induced by coupling to plasmons in near-field regime turns out to be significantly larger than for coupling of electrons to planarwave photons. This is of practical importance for enhancement of thin-film solar cell efficiency, both for semiconductor type (like III-V semiconductor based cells) and for
\end{abstract}


conjugate-polymer-based or dye organic plastic cells, intensively developed at present. We have described also a non-dissipative collective mode of surface plasmons in a chain of near-field-coupled metallic nanospheres, for particular size, separation parameters and wave-lengths. This would find an application in sub-diffraction electro-photonic circuit arrangement and for possible energy transport in solar cells, in particular in organic materials with low mobility of carriers.

PACS numbers: 73.21.-b, 36.40.Gk, 73.20.Mf, 78.67.Bf

Submitted to: J. Phys. D: Appl. Phys. 


\section{Introduction}

Rapid progress in plasmonics [1] (taking advantage of peculiar properties of plasmonpolaritons [2] in nano-structured metallic interfaces) and plasmonic applications in photonics and microelectronics [3] have focused attention on metallic modified systems in nano-scale and on collective excitations of metallic plasma in a confined geometry. Of particular interest are the recently reported experimental data on giant enhancement of photoluminescence and absorption of light by semiconductor surfaces (of photo-diodes) covered with metallic (gold, silver, or copper) nanospheres, with sphere radii of the order of several to several tens of nanometres $[4,5,6,7,8,9]$. These phenomena are recognized as promising for the enhancement of the efficiency of solar cells by the application of special metallic nanoparticle coverings of photo-active layers [4, 5]. Metallic nanospheres (or nanoparticles of other shape) can act as light converters, collecting energy of incident photons in surface plasmon oscillations. This energy can be next transferred to the semiconductor substrate in a more efficient manner in comparison to the direct photoeffect.

Experimental observations $[4,5,6,7,8,9]$ suggest, that the short range coupling between plasmons in nanospheres and electrons in the semiconductor substrate allows for significant growth of selective light energy transformation into a photo-current in the diode system. This phenomenon is not described in detail as of yet and moreover, some competitive mechanisms apparently contribute. Nevertheless, one can argue generally, that due to the nano-scale size of the metallic components, the momentum is not conserved, which leads to the allowance of all indirect optical interband transitions in the semiconductor layer, resulting in enhancement of the photo-current in comparison to the ordinary photo-effect, when only direct interband transitions are admitted.

The surface plasmons were originally considered by Mie [10] in 1908, who formulated a classical description of oscillations of electrical charge on the surface of the metallic sphere. The classical Mie frequencies are not dependent on the sphere radius, in contrast to the experimental observations for both small metallic clusters and larger spheres. Plasmon excitations in small metallic clusters (up to $2 \mathrm{~nm}$ ) were widely analyzed $[11,12,13,14]$ within mostly numerical calculations 'ab initio', including the shellmodel and the Kohn-Sham 'local density approximation' (LDA), limited, however, by numerical barriers to a few hundreds of electrons only. The emerging of the Mie response from the more general behaviour was presented $[11,12,13]$. Numerical analyses revealed the red-shift of Mie resonance mainly due to so-called spill-out of the electron cloud beyond the jellium rim. This effect is, however, not important for particles with radii larger than $2 \mathrm{~nm}$, and for nanospheres, of size of $10-60 \mathrm{~nm}$, the other effects are responsible for size-dependent shifts of resonances, pronounced in large particles and opposite to the red shift for small and ultra-small clusters.

The main factor for plasmon oscillations in large nanospheres was identified $[15,16,17]$ as radiation phenomena, which for radius larger than $10 \mathrm{~nm}$ dominate plasmon damping as growing with the radius $a$ as $a^{3}$. The pronounced cross-over in the 
vicinity of $10 \mathrm{~nm}$ (for $\mathrm{Au}, \mathrm{Ag}$ and $\mathrm{Cu}$ ) has been predicted [16] - for smaller nanospheres, the scattering damping (including boundaries and so-called Landau damping [18]) is important, while for larger spheres, the radiation losses are overwhelming. It is thus clear that rather large nanoparticles would play a role in all the phenomena linked with possible energy and information transport employing plasmon radiation, which well corresponds to experimental observations $[4,5,6,7,8,9]$.

In the present paper we develop the RPA (random phase approximation) semiclassical theory of plasmons, formulated [15] for large metallic nanospheres in analogy to RPA theory of plasmons in bulk metal $[19,20]$ (Pines-Bohm theory). The advantage of this description $[15,16]$ consists in full-analytic formulation allowing for application to more complicated physical situations, as e.g., plasmons interaction with surrounding medium, in order to describe plasmonic enhancement of photo-voltaic effect in the case of metallic particles deposited on the photo-active layer of the semiconductor, or for description of transport of plasmon-polaritons, with wide potential applications for subdiffraction nano-photonics [21]. It is indicated that the properties of plasmon oscillations in large nanospheres (i.e., with radii in the range of $10-60 \mathrm{~nm}$ ) are governed by strong radiation-induced energy losses, not analysed previously for such sized metallic particles, despite wide plasmon descriptions e.g., within fully classical Mie-type approach to large metallic particles $[22,23,24]$ and microscopic Kohn-Sham-type analyses of small and ultra-small metallic clusters $[11,12,18]$. For large nanospheres we have described a sharp crossover of radius dependence of resonant energy red-shift at ca $10 \mathrm{~nm}$ (for $\mathrm{Ag}$ and $\mathrm{Au}$ ), corresponding to competition of opposite resonance shifts due to scattering and irradiation losses, which has been confirmed by experimental observations in the case of dielectric surroundings of particles (in water colloidal solutions) [16]. The coupling of surface plasmons in the near-field zone with another systems, like a semiconductor substrate or other metallic nanosphere in the chain, is the main topic of the present consideration. An assessment of the energy transfer via this channel, as made in the present paper within the Fermi golden rule scheme, reveals a high efficiency of plasmonmediated energy transport and explains the experimentally observed PV efficiency growth of solar cell setups with metallic surface nano-modifications. It seems to be of a particular significance for thin film semiconductor solar cell technology including conjugated polymer semiconductor photo-active matrices with a possible commercial usage, provided an increase of their efficiency by relatively not-costly modifications, e.g., by sparse coverings of photo-active surface with noble metal nanoparticles, which is feasible in various techniques.

The paper is organized as follows. In the next section a short review of the model is given including results of analyses both for volume and surface plasmon excitations in spherical geometry. Damping phenomena with particular role of radiation losses for large nanospheres is described in the following section, in terms of the Lorentz friction. The separate discussion is addressed to giant enhancement of photo-voltaic effect by plasmon mediation, basing on the hypothesis that this growth corresponds to contribution of all indirect inter-band transitions in the substrate semiconductor, allowed 
in the nanostructure with near-field coupling (which is not a translation invariant system and thus without the momentum conservation condition imposed). The comparison with experimental data related to radiation of plasmons in metallic nanospheres is also given. In the last section, the undamped mode of collective surface plasmon excitation along the metallic nanosphere chain is demonstrated, in a correspondence with previous numerical simulations of long range plasmon-polariton modes [25].

\section{Surface and volume plasmons in large metallic nanospheres}

\subsection{RPA equation for collective electron oscillations in confined geometry}

We consider a metallic sphere with radius $a$ in a vacuum, $\varepsilon=1, \mu=1$. We assume that the interaction between electrons and ions is described by a local and weak pseudopotential (this condition corresponds to the so-called 'simple metal' case) [19], as e.g. for noble metals; of a particular significance are gold, silver and also copper nanoparticles due to the strong visible-light plasmon resonances in these materials. The Hamiltonian for this system has the form:

$$
\begin{aligned}
& \hat{H}=-\sum_{\nu=1}^{N} \frac{\hbar^{2} \nabla_{\nu}^{2}}{2 M}+\frac{1}{2} \sum_{\nu \neq \nu^{\prime}} u\left(\boldsymbol{R}_{\nu}-\boldsymbol{R}_{\nu^{\prime}}\right)-\sum_{j=1}^{N_{e}} \frac{\hbar^{2} \nabla_{j}^{2}}{2 m}+\frac{1}{2} \sum_{j \neq j^{\prime}} \frac{e^{2}}{\left|\boldsymbol{r}_{j}-\boldsymbol{r}_{j^{\prime}}\right|} \\
& +\sum_{\nu, j} w\left(\boldsymbol{R}_{\nu}-\boldsymbol{r}_{j}\right),
\end{aligned}
$$

where $\boldsymbol{R}_{\nu}, \boldsymbol{r}_{j}$ and $M, m$ are the positions and masses of the ions and electrons, respectively; $N$ is the number of ions in the sphere, $N_{e}=Z N$ is the number of collective electrons, $u\left(\boldsymbol{R}_{\nu}-\boldsymbol{R}_{\nu^{\prime}}\right)$ is the interaction of ions (ion is treated as a nucleus with electron core of closed shells) and $w\left(\boldsymbol{R}_{\nu}-\boldsymbol{r}_{j}\right)$ is the local pseudopotential of electron-ion interaction. Assuming the jellium model $[11,22,26]$ one can write for the background ion charge uniformly distributed over the sphere: $n_{e}(\boldsymbol{r})=n_{e} \Theta(a-r)$, where $n_{e}=N_{e} / V$ and $n_{e}|e|$ is the averaged positive charge density, $V=\frac{4 \pi a^{3}}{3}$ is the sphere volume and $\Theta$ is the Heaviside step-function.

A local electron density can be written as follows [19, 20]:

$$
\rho(\boldsymbol{r}, t)=<\Psi_{e}(t)\left|\sum_{j} \delta\left(\boldsymbol{r}-\boldsymbol{r}_{j}\right)\right| \Psi_{e}(t)>,
$$

with the Fourier picture:

$$
\tilde{\rho}(\boldsymbol{k}, t)=\int \rho(\boldsymbol{r}, t) e^{-i \boldsymbol{k} \cdot \boldsymbol{r}} d^{3} r=<\Psi_{e}(t)|\hat{\rho}(\boldsymbol{k})| \Psi_{e}(t)>,
$$

where the 'operator' $\hat{\rho}(\boldsymbol{k})=\sum_{j} e^{-i \boldsymbol{k} \cdot \boldsymbol{r}_{j}}$.

Using the above notation one can rewrite the electron part of the Hamiltonian (1), $\hat{H}_{e}$, in the following form [15]:

$$
\begin{aligned}
& \hat{H}_{e}=\sum_{j=1}^{N_{e}}\left[-\frac{\hbar^{2} \nabla_{j}^{2}}{2 m}\right]-\frac{e^{2}}{(2 \pi)^{3}} \int d^{3} k \tilde{n}_{e}(\boldsymbol{k}) \frac{2 \pi}{k^{2}}\left(\hat{\rho^{+}}(\boldsymbol{k})+\hat{\rho}(\boldsymbol{k})\right) \\
& +\frac{e^{2}}{(2 \pi)^{3}} \int d^{3} k \frac{2 \pi}{k^{2}}\left[\hat{\rho^{+}}(\boldsymbol{k}) \hat{\rho}(\boldsymbol{k})-N_{e}\right],
\end{aligned}
$$

where: $\tilde{n}_{e}(\boldsymbol{k})=\int d^{3} r n_{e}(\boldsymbol{r}) e^{-i \boldsymbol{k} \cdot \boldsymbol{r}}, \frac{4 \pi}{k^{2}}=\int d^{3} r \frac{1}{r} e^{-i \boldsymbol{k} \cdot \boldsymbol{r}}$. 
The motion equation has the form:

$$
\frac{d^{2} \hat{\rho}(\boldsymbol{k})}{d t^{2}}=\frac{1}{(i \hbar)^{2}}\left[\left[\hat{\rho}(\boldsymbol{k}), \hat{H}_{e}\right], \hat{H}_{e}\right] .
$$

Within the RPA and for Thomas-Fermi averaged kinetic energy formula [19], Eq. (5) attains the form $[15]$ :

$$
\begin{aligned}
& \frac{\partial^{2} \delta \tilde{\rho}(\boldsymbol{r}, t)}{\partial t^{2}}=\left[\frac{2}{3} \frac{\epsilon_{F}}{m} \nabla^{2} \delta \tilde{\rho}(\boldsymbol{r}, t)-\omega_{p}^{2} \delta \tilde{\rho}(\boldsymbol{r}, t)\right] \Theta(a-r) \\
& -\frac{2}{3 m} \nabla\left\{\left[\frac{3}{5} \epsilon_{F} n_{e}+\epsilon_{F} \delta \tilde{\rho}(\boldsymbol{r}, t)\right] \frac{\boldsymbol{r}}{r} \delta(a-r)\right\} \\
& -\left[\frac{2}{3} \frac{\epsilon_{F}}{m} \frac{\boldsymbol{r}}{r} \nabla \delta \tilde{\rho}(\boldsymbol{r}, t)+\frac{\omega_{p}^{2}}{4 \pi} \frac{\boldsymbol{r}}{r} \nabla \int d^{3} r_{1} \frac{1}{\left|\boldsymbol{r}-\boldsymbol{r}_{1}\right|} \delta \tilde{\rho}\left(\boldsymbol{r}_{1}, t\right)\right] \delta(a-r) .
\end{aligned}
$$

In the above formula $\omega_{p}$ is the bulk plasmon frequency, $\omega_{p}^{2}=\frac{4 \pi n_{e} e^{2}}{m}$ (it was taken into account that $\left.\nabla \Theta(a-r)=-\frac{\boldsymbol{r}}{r} \delta(a-r)\right)$. The solution of Eq. (6) can be decomposed into two parts related to the distinct domains:

$$
\delta \tilde{\rho}(\boldsymbol{r}, t)=\left\{\begin{array}{l}
\delta \tilde{\rho}_{1}(\boldsymbol{r}, t), \text { for } r<a, \\
\delta \tilde{\rho}_{2}(\boldsymbol{r}, t), \text { for } r \geq a,(r \rightarrow a+),
\end{array}\right.
$$

corresponding to the volume and surface excitations, respectively. These two parts of local electron density fluctuations satisfy the equations (according to Eq. (6)):

$$
\frac{\partial^{2} \delta \tilde{\rho}_{1}(\boldsymbol{r}, t)}{\partial t^{2}}=\frac{2}{3} \frac{\epsilon_{F}}{m} \nabla^{2} \delta \tilde{\rho}_{1}(\boldsymbol{r}, t)-\omega_{p}^{2} \delta \tilde{\rho}_{1}(\boldsymbol{r}, t),
$$

and (here $\epsilon=0+$ )

$$
\begin{aligned}
& \frac{\partial^{2} \delta \tilde{\rho}_{2}(\boldsymbol{r}, t)}{\partial t^{2}}=-\frac{2}{3 m} \nabla\left\{\left[\frac{3}{5} \epsilon_{F} n_{e}+\epsilon_{F} \delta \tilde{\rho}_{2}(\boldsymbol{r}, t)\right] \frac{\boldsymbol{r}}{r} \delta(a+\epsilon-r)\right\} \\
& -\left[\frac{2}{3} \frac{\epsilon_{F}}{m} \frac{\boldsymbol{r}}{r} \nabla \delta \tilde{\rho}_{2}(\boldsymbol{r}, t)+\frac{\omega_{p}^{2}}{4 \pi} \frac{\boldsymbol{r}}{r} \nabla \int d^{3} r_{1} \frac{1}{\left|\boldsymbol{r}-\boldsymbol{r}_{1}\right|}\left(\delta \tilde{\rho}_{1}\left(\boldsymbol{r}_{1}, t\right) \Theta\left(a-r_{1}\right)\right.\right. \\
& \left.\left.+\delta \tilde{\rho}_{2}\left(\boldsymbol{r}_{1}, t\right) \Theta\left(r_{1}-a\right)\right)\right] \delta(a+\epsilon-r) .
\end{aligned}
$$

\subsection{Solutions of plasmon RPA equations}

Eqs (8) and (9) can be solved upon imposed boundary and symmetry conditions. Let us represent both parts of the electron fluctuation in the following manner:

$$
\begin{aligned}
& \delta \tilde{\rho}_{1}(\boldsymbol{r}, t)=n_{e}\left[f_{1}(r)+F(\boldsymbol{r}, t)\right], \text { for } r<a, \\
& \delta \tilde{\rho}_{2}(\boldsymbol{r}, t)=n_{e} f_{2}(r)+\sigma(\Omega, t) \delta(r+\epsilon-a), \epsilon=0+, \text { for } r \geq a,(r \rightarrow a+),
\end{aligned}
$$

and now let us choose the convenient initial conditions, $\left.F(\boldsymbol{r}, t)\right|_{t=0}=0,\left.\sigma(\Omega, t)\right|_{t=0}=0$, ( $\Omega$ is the spherical angle), moreover $\left.\left(1+f_{1}(r)\right)\right|_{r=a}=\left.f_{2}(r)\right|_{r=a}$ (continuity condition), $\left.F(\boldsymbol{r}, t)\right|_{r \rightarrow a}=0, \int \rho(\boldsymbol{r}, t) d^{3} r=N_{e}$ (neutrality condition).

We arrive $[15,16]$ thus with the explicit form of the solutions of Eqs $(8,9)$ :

$$
\begin{aligned}
& f_{1}(r)=-\frac{k_{T} a+1}{2} e^{-k_{T}(a-r) \frac{1-e^{-2 k_{T} r}}{k_{T} r}}, \text { for } r<a, \\
& f_{2}(r)=\left[k_{T} a-\frac{k_{T} a+1}{2}\left(1-e^{-2 k_{T} a}\right)\right] \frac{e^{-k_{T}(r-a)}}{k_{T} r}, \text { for } r \geq a,
\end{aligned}
$$

where $k_{T}=\sqrt{\frac{6 \pi n_{e} e^{2}}{\epsilon_{F}}}=\sqrt{\frac{3 \omega_{p}^{2}}{v_{F}^{2}}}$ (Thomas-Fermi inverse radius). For the time-dependent parts of the electron fluctuations we find $[15,17]$ :

$$
F(\boldsymbol{r}, t)=\sum_{l=1}^{\infty} \sum_{m=-l}^{l} \sum_{n=1}^{\infty} A_{l m n} j_{l}\left(k_{n l} r\right) Y_{l m}(\Omega) \sin \left(\omega_{n l} t\right),
$$


and

$$
\begin{aligned}
& \sigma(\Omega, t)=\sum_{l=1}^{\infty} \sum_{m=-l}^{l} \frac{B_{l m}}{a^{2}} Y_{l m}(\Omega) \sin \left(\omega_{0 l} t\right) \\
& +\sum_{l=1}^{\infty} \sum_{m=-l}^{l} \sum_{n=1}^{\infty} A_{l m n} \frac{(l+1) \omega_{p}^{2}}{l \omega_{p}^{2}-(2 l+1) \omega_{n l}^{2}} Y_{l m}(\Omega) n_{e} \int_{0}^{a} d r_{1} \frac{r_{l}^{l+2}}{a^{l+2}} j_{l}\left(k_{n l} r_{1}\right) \sin \left(\omega_{n l} t\right),
\end{aligned}
$$

where $j_{l}(\xi)=\sqrt{\frac{\pi}{2 \xi}} I_{l+1 / 2}(\xi)$ is the spherical Bessel function, $Y_{l m}(\Omega)$ is the spherical

function, $\omega_{n l}=\omega_{p} \sqrt{1+\frac{x_{n l}^{2}}{k_{T}^{2} a^{2}}}$ are the frequencies of electron volume self-oscillations (volume plasmon frequencies), $x_{n l}$ are the nodes of the Bessel function $j_{l}(\xi), k_{n l}=x_{n l} / a$, $\omega_{0 l}=\omega_{p} \sqrt{\frac{l}{2 l+1}}$ are the frequencies of electron surface self-oscillations (surface plasmon frequencies).

From the above equations it follows thus that the local electron density (within RPA attitude) has the form:

$$
\rho(\boldsymbol{r}, t)=\rho_{0}(r)+\rho_{n e q}(\boldsymbol{r}, t),
$$

where the RPA equilibrium electron distribution is (correcting the uniform distribution $\left.n_{e}\right)$ :

$$
\rho_{0}(r)=\left\{\begin{array}{l}
n_{e}\left[1+f_{1}(r)\right], \text { for } r<a, \\
n_{e} f_{2}(r), \text { for } r \geq a, r \rightarrow a+
\end{array}\right.
$$

and the nonequilibrium, of plasmon oscillation type, is:

$$
\rho_{\text {neq }}(\boldsymbol{r}, t)=\left\{\begin{array}{l}
n_{e} F(\boldsymbol{r}, t), \text { for } r<a, \\
\sigma(\Omega, t) \delta(a+\epsilon-r), \epsilon=0+, \text { for } r \geq a, r \rightarrow a+.
\end{array}\right.
$$

The function $F(\boldsymbol{r}, t)$ displays volume plasmon oscillations, while $\sigma(\Omega, t)$ describes the surface plasmon oscillations. Let us emphasize that in the formula for $\sigma(\Omega, t)$, Eq. (13), the first term corresponds to surface self-oscillations, while the second term describes the surface oscillations induced by the volume plasmons. The frequencies of the surface self-oscillations are

$$
\omega_{0 l}=\omega_{p} \sqrt{\frac{l}{2 l+1}},
$$

which, for $l=1$, agrees with the dipole type surface oscillations described originally by Mie [10], $\omega_{01}=\omega_{p} / \sqrt{3}$ (for simplicity, denoted hereafter as $\omega_{1}=\omega_{01}$ ).

\section{Damping of plasmons in large nanospheres}

One can take advantage of the oscillator form of RPA semiclassical equations for both volume and surface modes of plasmons in finite geometry, and can phenomenologically include damping of plasmons in analogy to oscillator damping. Let us also assume that both volume and surface plasmon oscillations are damped with the time ratio $\tau_{0}$ which can be phenomenologically accounted for via the additional term, $-\frac{2}{\tau_{0}} \frac{\partial \delta \rho(\boldsymbol{r}, t)}{\partial t}$, to the right-hand-side of above equations. They attain the form:

$$
\frac{\partial^{2} \delta \rho_{1}(\boldsymbol{r}, t)}{\partial t^{2}}+\frac{2}{\tau_{0}} \frac{\partial \delta \rho_{1}(\boldsymbol{r}, t)}{\partial t}=\frac{2}{3} \frac{\epsilon_{F}}{m} \nabla^{2} \delta \rho_{1}(\boldsymbol{r}, t)-\omega_{p}^{2} \delta \rho_{1}(\boldsymbol{r}, t)
$$


and

$$
\begin{aligned}
& \frac{\partial^{2} \delta \rho_{2}(\boldsymbol{r}, t)}{\partial t^{2}}+\frac{2}{\tau_{0}} \frac{\partial \delta \rho_{2}(\boldsymbol{r}, t)}{\partial t}=-\frac{2}{3 m} \nabla\left\{\left[\frac{3}{5} \epsilon_{F} n_{e}+\epsilon_{F} \delta \rho_{2}(\boldsymbol{r}, t)\right] \frac{\boldsymbol{r}}{r} \delta(a+\epsilon-r)\right\} \\
& -\left[\frac{2}{3} \frac{\epsilon_{F}}{m} \frac{\boldsymbol{r}}{r} \nabla \delta \rho_{2}(\boldsymbol{r}, t)+\frac{\omega_{p}^{2}}{4 \pi} \frac{\boldsymbol{r}}{r} \nabla \int d^{3} r_{1} \frac{1}{\left|\boldsymbol{r}_{-} \boldsymbol{r}_{1}\right|}\left(\delta \rho_{1}\left(\boldsymbol{r}_{1}, t\right) \Theta\left(a-r_{1}\right)\right.\right. \\
& \left.\left.+\frac{1}{\varepsilon} \delta \rho_{2}\left(\boldsymbol{r}_{1}, t\right) \Theta\left(r_{1}-a\right)\right)+\frac{e n_{e}}{m} \frac{\boldsymbol{r}}{r} \cdot \boldsymbol{E}(t)\right] \delta(a+\epsilon-r) .
\end{aligned}
$$

For the homogeneous forcing field $\boldsymbol{E}(t)$, only dipole surface mode can be excited and the electron dynamics resolves to the equation for a single dipole type mode, described by the function $Q_{1 m}(t)$. The function $Q_{1 m}(t)$ satisfies the equation:

$$
\begin{aligned}
& \frac{\partial^{2} Q_{1 m}(t)}{\partial t^{2}}+\frac{2}{\tau_{0}} \frac{\partial Q_{1 m}(t)}{\partial t}+\omega_{1}^{2} Q_{1 m}(t) \\
& =\sqrt{\frac{4 \pi}{3}} \frac{e n_{e}}{m}\left[E_{z}(t) \delta_{m 0}+\sqrt{2}\left(E_{x}(t) \delta_{m 1}+E_{y}(t) \delta_{m-1}\right)\right],
\end{aligned}
$$

where $\omega_{1}=\omega_{01}=\frac{\omega_{p}}{\sqrt{3 \varepsilon}}$ (it is a dipole-type surface plasmon Mie frequency [10]). Only this function contributes to the dynamical response to the homogeneous electric field. Thus for the homogeneous forcing field, electron density fluctuations:

$$
\delta \rho(\boldsymbol{r}, t)=\left\{\begin{array}{l}
0, \text { for } r<a, \\
\sum_{m=-1}^{1} Q_{1 m}(t) Y_{1 m}(\Omega) \text { for } r \geq a, r \rightarrow a+.
\end{array}\right.
$$

In general, $F(\boldsymbol{r}, t)$ (volume plasmons) and $\sigma(\Omega, t)$ (surface plasmons) contribute to plasmon oscillations. Nevertheless, in the case of homogeneous perturbation, only the surface $l=1$ mode is excited (it is a case of plasmon exciting by the visible light, when for nanospheres with radius of $10-100 \mathrm{~nm}$, the dipole approximation holds).

For plasmon oscillations given by Eq. (21) one can calculate the corresponding dipole,

$$
\boldsymbol{D}(t)=e \int d^{3} r \boldsymbol{r} \delta \rho(\boldsymbol{r}, t)=\frac{4 \pi}{3} e \boldsymbol{q}(t) a^{3},
$$

where $Q_{11}(t)=\sqrt{\frac{8 \pi}{3}} q_{x}(t), Q_{1-1}(t)=\sqrt{\frac{8 \pi}{3}} q_{y}(t), Q_{10}(t)=\sqrt{\frac{4 \pi}{3}} q_{x}(t)$ and $\boldsymbol{q}(t)$ satisfies the equation (cf. Eq. (20)),

$$
\left[\frac{\partial^{2}}{\partial t^{2}}+\frac{2}{\tau_{0}} \frac{\partial}{\partial t}+\omega_{1}^{2}\right] \boldsymbol{q}(t)=\frac{e n_{e}}{m} \boldsymbol{E}(t) .
$$

There are various mechanisms of plasmon damping, which could be effectively accounted for via phenomenological oscillator type damping term. All types of scattering phenomena, including electron-electron and electron-phonon interactions, as well contribution of boundary scattering effect [21] cause significant attenuation of plasmons in particular in small metal clusters. All these contributions to damping time ratio scale as $\frac{1}{a}$ and are of lowering significance with the radius growth. In the following subsection we argue that damping of plasmons caused by radiation losses scales conversely, as $a^{3}$, and for large nanospheres this channel dominates plasmon attenuation. 


\subsection{Lorentz friction for plasmons}

The nanosphere surface plasmons can be induced by a homogeneous electric field [16], while the volume mode excitation needs field inhomogeneity on the radius scale (and therefore the visible light cannot excite volume modes in the nanospheres with radii of $10-60 \mathrm{~nm})$. Plasmon oscillations are themselves a source of the e-m radiation. This radiation takes away the energy of plasmons resulting in their damping, which can be described as the Lorentz friction [27]. This damping was not included in $\tau_{0}$ in Eq. (23).

Various processes contribute to the attenuation of plasmon oscillations in the case of metallic nanospheres. The most important (and thoroughly analyzed in the case of small and ultra-small metallic cluster, for which they play much more pronounced role in comparison to large nanospheres) are scattering of electrons on other electrons, on defects, on phonons and on nanoparticle boundary - all they lead to damping rate expressed by the simplified formula [21]:

$$
\frac{1}{\tau_{0}} \simeq \frac{v_{F}}{2 \lambda_{B}}+\frac{c v_{F}}{2 a}
$$

where, $C$ is the constant of unity order, $a$ is the nanosphere radius, $v_{F}$ is the Fermi velocity in metal, $\lambda_{B}$ is the electron free path in bulk (including scattering of electrons on other electrons, on impurities and on phonons, and its inverse ratio to Fermi velocity determines the time scale of decay of any electron-built states, also of plasmons [21]); for $\mathrm{Ag}, v_{F}=1.4 \times 10^{6} \mathrm{~m} / \mathrm{s}$ and $\lambda_{B} \simeq 57 \mathrm{~nm}$ (at room temperature); the latter term in the formula (24) accounts for scattering of electrons on the boundary of the nanoparticle, while the former one corresponds to scattering processes similar as in bulk. The other effects, as the so-called Landau damping (especially important in small clusters [18, 28]), corresponding to decay of plasmon for high energy particle-hole pair, are of lowering significance for nanosphere radii larger than $2-3 \mathrm{~nm}$ [18] and completely negligible for radii larger than $10 \mathrm{~nm}$. Note that the similarly lowering role with the radius growth plays also electron liquid spill-out effect $[11,12]$, though it was of primary importance for small clusters $[11,14]$.

The e-m wave emission which causes electron friction can be described as the additional electric field [27],

$$
\boldsymbol{E}_{L}=\frac{2}{3 \varepsilon^{3 / 2} v^{3}} \frac{\partial^{3} \boldsymbol{D}(t)}{\partial t^{3}}
$$

where $v=\frac{c}{\sqrt{\varepsilon}}$ is the light velocity in the dielectric medium, and $\boldsymbol{D}(t)$ is the dipole of the nanosphere. According to Eq. (22) we arrive at the following:

$$
\boldsymbol{E}_{L}=\frac{2 e}{3 \varepsilon v^{2}} \frac{4 \pi}{3} a^{3} \frac{\partial^{3} \boldsymbol{q}(t)}{\partial t^{3}} .
$$

Substituting this into Eq. (23), we get

$$
\left[\frac{\partial^{2}}{\partial t^{2}}+\frac{2}{\tau_{0}} \frac{\partial}{\partial t}+\omega_{1}^{2}\right] \boldsymbol{q}(t)=\frac{e n_{e}}{m} \boldsymbol{E}(t)+\frac{2}{3 \omega_{1}}\left(\frac{\omega_{1} a}{v}\right)^{3} \frac{\partial^{3} \boldsymbol{q}(t)}{\partial t^{3}} .
$$


If one rewrites the above equation (for $\boldsymbol{E}=0$ ) in the form

$$
\left[\frac{\partial^{2}}{\partial t^{2}}+\omega_{1}^{2}\right] \boldsymbol{q}(t)=\frac{\partial}{\partial t}\left[-\frac{2}{\tau_{0}}+\frac{2}{3 \omega_{1}}\left(\frac{\omega_{1} a}{v}\right)^{3} \frac{\partial^{2} \boldsymbol{q}(t)}{\partial t^{2}}\right],
$$

thus, one notes that the zeroth order approximation (neglecting attenuation) corresponds to the equation:

$$
\left[\frac{\partial^{2}}{\partial t^{2}}+\omega_{1}^{2}\right] \boldsymbol{q}(t)=0 .
$$

In order to solve Eq. (28) in the next step of perturbation iteration, one can substitute, in the right-hand-side of this equation, $\frac{\partial^{2} \boldsymbol{q}(t)}{\partial t^{2}}$ by $-\omega_{1}^{2} \boldsymbol{q}(t)$ (acc. to Eq. (29)).

Therefore, if one assumes the above estimation, $\frac{\partial^{3} \boldsymbol{q}(t)}{\partial t^{3}} \simeq-\omega_{1}^{2} \frac{\partial \boldsymbol{q}(t)}{\partial t}$, one can include the Lorentz friction in a renormalised damping term:

$$
\left[\frac{\partial^{2}}{\partial t^{2}}+\frac{2}{\tau} \frac{\partial}{\partial t}+\omega_{1}^{2}\right] \boldsymbol{q}(t)=\frac{e n_{e}}{m} \boldsymbol{E}(t),
$$

where

$$
\frac{1}{\tau}=\frac{1}{\tau_{0}}+\frac{\omega_{1}}{3}\left(\frac{\omega_{1} a}{v}\right)^{3} \simeq \frac{v_{F}}{2 \lambda_{B}}+\frac{C v_{F}}{2 a}+\frac{\omega_{1}}{3}\left(\frac{\omega_{1} a}{v}\right)^{3},
$$

where we used for $\frac{1}{\tau_{0}} \simeq \frac{v_{F}}{2 \lambda_{B}}+\frac{C v_{F}}{2 a},\left(\lambda_{B}\right.$ is the free path in bulk, $v_{F}$ is the Fermi velocity, and $C \simeq 1$ is a constant) [21], which corresponds to inclusion of plasmon damping due to electron scattering on other electrons and on the nanoparticle boundary. Renormalised damping causes a change in the shift of self-frequencies of free surface plasmons, $\omega_{1}^{\prime}=\sqrt{\omega_{1}^{2}-\frac{1}{\tau^{2}}}$.

The resulting cross-over is depicted in Fig. 1. Using Eq. (31) one can determine the radius $a_{0}$ corresponding to a minimal damping,

$$
a_{0}=\frac{\sqrt{3}}{\omega_{p}}\left(v_{F} c^{3} \sqrt{\varepsilon} / 2\right)^{1 / 4} .
$$

Note, that the Lorentz friction, being of the third order with respect to timeretardation shift [27], includes the retardation effects via plasmon damping induced by irradiation [29]. The other channel of plasmon damping corresponds to nonlocal effects including scattering on the nanoparticle surface [30]. We have demonstrated that the retardation and the nonlocal effects lead to opposite, red and blue shifts of the resonance frequency [29]. This strong cross-over of plasmon damping is the most significant property of plasmon radiation from nanospheres, which decides on particular applications related with plasmon radiative transport of energy and information.

Note also, that one can verify [16] the above calculated Lorentz friction contribution to plasmon damping by estimation of the energy transfer in the far-field region (which can be expressed by the Poynting vector) and via comparison with the energy loss of plasmon oscillation. We have arrived $[15,16]$ at the same formula for damping time rate as given by Eq. (30). The radius dependent shift of the resonance resulting due to strong irradiation-induced plasmon damping was verified also experimentally [16] by measurement of light extinction in colloidal solutions of nanoparticles with different size 

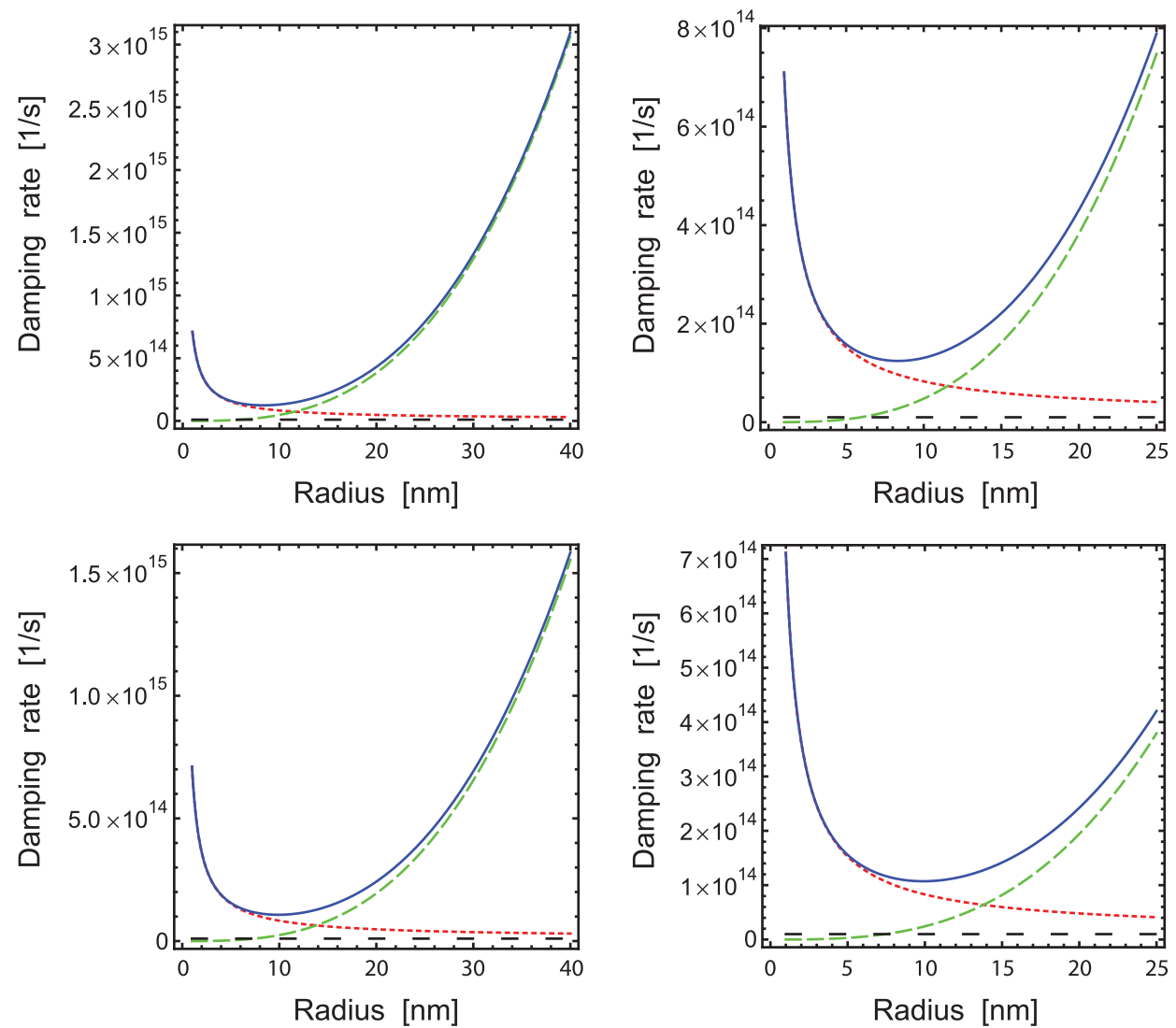

Figure 1. (Colour online) Upper: the damping rate with respect to the sphere radius $a$ in the region of cross-over for $\mathrm{Ag}$ nanospheres (in water colloidal solution): dotted line - contribution of scattering $\sim \frac{v_{F}}{\lambda_{B}}+\frac{v_{F}}{a}$, dashed line - contribution of radiation in far-field $\sim a^{3}$, solid line - the sum of both contributions (dashed horizontal line-level $10^{13} 1 / \mathrm{s}$ ); bottom: the damping rate with respect to the sphere radius in the region of cross-over for Au nanospheres (in water colloidal solution): dotted line - contribution of scattering, dashed line - contribution of radiation in far-field, solid line - sum of both contributions

(it is done [16] for $\mathrm{Au}, 10-80 \mathrm{~nm}$, and $\mathrm{Ag}, 10-60 \mathrm{~nm}$ ). These measurements clearly support the $a^{3}$ plasmon damping behaviour, as described above for the far-field zone radiation losses in a dielectric surroundings.

In large nanospheres the overwhelming role for decay of plasmons play radiation losses, which scale as $a^{3}$ with nanosphere radius (in the range $10-60 \mathrm{~nm}$ for $a$ ) and give rise to a pronounced cross-over of plasmon damping versus nanosphere size at ca $10 \mathrm{~nm}$ of radius (for $\mathrm{Au}$ and $\mathrm{Ag}$ ) - cf. Fig. 1. It is, however, an important question of ultimate damping behaviour with further radius growth. As it follows from our analysis, the cubic-type enhancement of attenuation rate with radius growth quickly saturates at ca $50-60 \mathrm{~nm}$, due to nonlinear (in sense of the time derivative order) contribution of the Lorentz friction [27]. Linearisation of the Lorentz friction resulted 
in the cubic radius dependence of attenuation rate and it was justified only for not high damping [16]. When, however, this attenuation attains the large value, the third order derivative correction quenches the further growth and damping saturates, for limiting large nanospheres (at radius of order of $50-80 \mathrm{~nm}$, as we have verified also experimentally for $\mathrm{Au}$ and $\mathrm{Ag}$ colloidal water solutions [16]). Thus for plasmon applications to irradiation energy transport the nanosphere size window $10-60 \mathrm{~nm}$ seems to be most convenient.

If, however, in the vicinity of the nanosphere the another system is located, the situation would change. For the case when the nanosphere is deposited on the semiconductor surface, the near-field coupling of plasmons with semiconductor band electrons must be included.

\subsection{Damping of plasmons due to near-field coupling with semiconductor band electrons}

Assuming stationary conditions (i.e., constant-in-time amplitude of the surface plasmon oscillations, which corresponds to a balance of the incoming energy of incident photons with the energy outgoing to the semiconductor substrate) the relevant damping is governed by the near-field dipole interaction (for $R \ll \lambda$ ) expressed by the scalar potential [27] with an amplitude $D_{0}(\omega)$,

$$
\varphi(\boldsymbol{R}, t)=\frac{1}{\varepsilon_{0} R^{2}} \boldsymbol{n} \cdot \boldsymbol{D}_{0}(\omega) \sin (\omega t) .
$$

The matrix element of near-field dipole interaction for the transition of a semiconductor electron from the state in the valence to the conduction band, assumed as $\Psi_{i(f)}(\boldsymbol{r}, t)=$ $(2 \pi)^{-3 / 2} \exp \left[i \boldsymbol{k} \cdot \boldsymbol{r}-i E_{i(f)}(\boldsymbol{k}) t / \hbar\right]$ ( $i$-initial, $f$-final, respectively) can be calculated by application of the Fermi golden rule, which leads to a probability of transition per time unit [15],

$$
\delta w=\frac{e^{2}\left(D_{0}(\omega)\right)^{2} \mu \sqrt{m_{p}^{*} m_{n}^{*}}}{3\left(4 \pi^{3}\right)^{2} \hbar^{5} \varepsilon^{2}}\left(\hbar \omega-E_{g}\right),
$$

where $D_{0}(\omega)$ is the surface plasmon dipole oscillation amplitude, adjusted to the balance of energy income and outcome (via the shift of the resonance for stationary driven and damped oscillations).

Taking into account that the number of incident photons in the volume $V$ of a semiconductor equals $\frac{\varepsilon E_{0}^{2} V}{8 \pi \hbar \omega}$ and that the volume rate of metallic components is $C_{0}=N_{m} \frac{4 \pi a^{3}}{3 V}\left(N_{m}\right.$ - the number of nanospheres), the probability that an energy of a single incident photon is transferred to the semiconductor via surface plasmons on metallic nano-admixtures can be expressed as (with $\delta w$ given by Eq. (34)):

$$
q_{m}=\beta N_{m} \delta w\left(\frac{\varepsilon E_{0}^{2} V}{8 \pi \hbar \omega}\right)^{-1}=\frac{\beta C_{0} e^{2} \omega f^{2}(\omega) a^{3}}{8 \pi^{6} \hbar^{4} \varepsilon} \mu \sqrt{m_{p}^{*} m_{n}^{*}}\left(\hbar \omega-E_{g}\right)
$$

where $f(\omega)=\frac{\omega_{1}^{2}}{\sqrt{\left(\omega_{1}^{2}-\omega^{2}\right)^{2}+4 \omega^{2} / \tau^{2}}}$ is the amplitude of forced surface plasmon oscillations, $\beta$ is an additional phenomenological factor accounting for proximity near-field contact reduction (via comparison with the experimental data, $\beta \sim 10^{-2}$ ) [15]. 
In order to assess efficiency of the near-field coupling channel one can estimate the ratio of the probability of energy absorption in the semiconductor via mediation of surface plasmons (per single photon incident on the metallic nanospheres) to the energy attenuation in the semiconductor directly from a planar wave illumination (also per single photon). In the latter case the energy attenuation in the semiconductor per single incident photon is given by the formula for ordinary photo-effect [31], $q=\frac{2 \sqrt{2}}{3 \pi^{6}} \frac{e^{2} \mu^{5 / 2}}{m_{p}^{* 2} \omega \varepsilon \hbar^{3}}\left(\hbar \omega-E_{g}\right)^{3 / 2}$. The ratio $\frac{q_{m}}{q}$ turns out to be of order of $10^{4} \frac{\beta 40}{H[n m]}$ (at a typical surface density of nanoparticles, $n_{s} \sim 10^{8} / \mathrm{cm}^{2}$ ) which (including the phenomenological factor $\beta$, and $H$-the semiconductor photo-active layer depth) is sufficient to explain the scale of the experimentally observed strong enhancement of absorption and emission rates.

The estimation of electron inter-band transition probability for the case of coupling of band electrons with near-field dipole radiation of plasmons in the nanosphere, as presented above, is idealized to an atomic limit. The strong enhancement of this transition probability is linked with allowance of momentum-non-conserved transitions. For an atomic limit the square of matrix element of perturbation between band states (as needed for a golden rule formula), $\left|<\boldsymbol{k}_{1}\right| \hat{w}\left|\boldsymbol{k}_{2}>\right|^{2} \sim \frac{1}{q^{2}}$, where $\boldsymbol{q}=\boldsymbol{k}_{2}-\boldsymbol{k}_{2}$ is the momentum transfer. It indicates that large $q$ do not give a significant contribution, though quenching of momentum-indirect transitions is not so restrictive as in the case of perturbation by a planar wave, resulting in $\delta(\boldsymbol{q})$ factor (in the ordinary photo-effect). For finite size nanospheres (with radius a) one can expect that the estimated above enhancement of interband transition probability of electrons in substrate semiconductor caused by near-field radiation of plasmons, and generated by momentum indirect transitions, would be of lower significance. The size dependent factor $\left(\frac{\sin (q a)}{q a}\right)^{2}$, multiplies the squared perturbation matrix element in this case, which finally results in the renormalisation of transition probability by the factor $\frac{1}{(a[n m])^{2}}$. For $a \sim 10-60$ $\mathrm{nm}$, this gives reducing of the transition probability by two-three orders, which we have already included by the effective phenmomenological factor $\beta$ (fitted form the experiment, $\left.\beta \simeq 28 \times 10^{-3}\left(\frac{50}{a[n m]}\right)^{2}\right)$. The enhancement of the near-field induced interband transition, in the case of large nanospheres, is, however, still significant as the reducing role of size-related quenching of transitions is partly compensated by $\sim a^{3}$ growth of the dipole amplitude of plasmon oscillations.

High efficiency (even if decreased by $\beta$ ) of the near-field energy transfer from surface plasmons to the semiconductor substrate is caused mainly by a contribution of all interband transitions, not restricted here to the direct (vertical) ones as for ordinary photo-effect, due to the absence of the momentum conservation constraints for nanosystems. The strengthening of the probability transition due to all indirect interband paths of excitations in the semiconductor is probably responsible for the observed experimentally strong enhancement of light absorption and emission in diode systems mediated by surface plasmons in nanoparticle surface coverings $[4,5,6,7,8,9]$. 
In the balanced state of the system, when the incoming energy of light is transferred to the semiconductor via near-field coupling, we deal with the stationary solution of driven and damped oscillator. The driving force is the electric field of the incident planar wave, and the damping force is the near-field energy transfer described by the $\frac{1}{\tau}$ (assuming that this dissipation channel is dominating). The resulting red-shifted resonance with simultaneously reduced amplitude allows for the accommodation to the balance of energy transfer to the semiconductor with incident photon energy. The amplitude of resonant plasmon oscillations $D_{0}(\omega)$ is thus shaped by $f(\omega)=\frac{1}{\sqrt{\left(\omega_{1}^{2}-\omega^{2}\right)^{2}+4 \omega^{2} / \tau^{2}}}$. The extremum of red-shifted resonance is attained at $\omega_{m}=\omega_{1} \sqrt{1-2\left(\omega_{1} \tau\right)^{-2}}$ with corresponding amplitude $\sim \tau /\left(2 \sqrt{\omega_{1}^{2}-\tau^{-2}}\right)$. This shift is proportional to $1 /\left(\omega_{1} \tau^{2}\right)$ and scales with nanosphere radius a similarly (diminishes with decreasing $a$ ) as in the experimental observations [7] (note again that for scatteringinduced $1 / \tau_{0}$ the dependence on $a$ is opposite [grows with decreasing $a$ ]).

In order to compare with the experiment let us estimate the photo-current in the case of a metallic modified surface in relation to the ordinary photo-effect. The photocurrent is given by $I^{\prime}=|e| N\left(q+q_{m}\right) A$, where $N$ is the number of incident photons and $q$ and $q_{m}$ are the probabilities of single photon attenuation in the ordinary photoeffect [31] and in that one enhanced due to the presence of metallic nanospheres, i.e., of $q=\frac{2 \sqrt{2}}{3 \pi^{6}} \frac{e^{2} \mu^{5 / 2}}{m_{p}^{* 2} \omega \varepsilon \hbar^{3}}\left(\hbar \omega-E_{g}\right)^{3 / 2}$ and $q_{m}$ given by Eq. (35); $A=\frac{\tau_{f}^{n}}{t_{n}}+\frac{\tau_{f}^{p}}{t_{p}}$ is the amplification factor $\left(\tau_{f}^{n(p)}\right.$ is the annihilation time of both sign carriers, $t_{n(p)}$ is the drive time for carriers [the time of traversing the distance between electrodes]). From the above formulae, it follows that (here $I=I^{\prime}\left(q_{m}=0\right)$, i.e., the photo-current without metallic modifications)

$$
\frac{I^{\prime}}{I}=1+7.95 \cdot 10^{5} c_{0} \frac{m_{p}^{*}}{m_{n}^{*}}\left(\frac{2 a}{100[1 \mathrm{~nm}]} \sqrt{\frac{\hbar \omega_{1}[\mathrm{eV}]}{x}\left(\frac{m_{p}^{*}}{m}+\frac{m_{n}^{*}}{m}\right)}\right)^{3} \phi(x),
$$

where $c_{0}=\frac{4 \pi a^{3}}{3} \beta \frac{n_{s}}{H}$, with $n_{s}$ as the surface density of metallic nanospheres, $H$ as the semiconductor layer depth, $\phi(x)=\frac{x^{2}}{\left(x^{2}-1\right)^{2}+4 x^{2} / x_{1}^{2}} \frac{1}{\sqrt{x-x_{g}}}, x=\omega / \omega_{1}, x_{1}=\tau \omega_{1}$, $x_{g}=E_{g} /\left(\hbar \omega_{1}\right), \hbar \omega_{1}=2.72 \mathrm{eV}, m_{n(p)}$ as the effective mass of conduction band and valence band carriers (for $S i, m_{n}^{*}=0.19(0.98) m$ and $m_{p}^{*}=0.16(0.52) m$, for light (heavy) carriers, band gap $\left.E_{g}=1.14 \mathrm{eV}, \varepsilon=12\right), m$ as the bare electron mass.

The results are summarized in Tab. 1 and in Fig. 2, for various radii of the nanospheres, and reproduce well the experimental behaviour [7]. By $x_{m}$ we denote the frequencies corresponding to the maximum value of the photo-current (i.e., to the maximum of $\left.I^{\prime} / I\right)$.

Both channels of photon absorption resulting in photo-current in the semiconductor sample are included, the direct ordinary photo-effect absorption with probability of transitions given by $q$ and the plasmon mediated absorption with probability $q_{m}$, respectively. Note also that some additional effects like reflection of the incident photons or destructive interference on metallic net would contribute and it was phenomenologically accounted for in the plasmon mediated channel by the experiment 
fitted factor $\beta$. These corrections are, however, rather not strong for the considered low densities of metallic coverings of order of $10^{8} / \mathrm{cm}^{2}$, and nanosphere sizes well lower than the resonant wave-length, though for larger concentrations and larger nanosphere sizes, would play a stronger reducing role [32, 33]. The resonance threshold was accounted for by the damped resonance envelope function $\phi(x)$ in Eq. (36) including also semiconductor band-gap limit.

Tab. 1. Comparison with the experimental data [7] for $\mathrm{Au} / \mathrm{Si}$

\begin{tabular}{|l|l|l|l|l|l|l|}
\hline$a[\mathrm{~nm}]$ & $\begin{array}{l}n_{s} \\
{\left[10^{8} / \mathrm{cm}^{2}\right]}\end{array}$ & $x_{m}$ & $\begin{array}{l}\omega_{m}=x_{m} \hbar \omega_{1} \\
(\text { theor})[\mathrm{eV}]\end{array}$ & $\begin{array}{l}\hbar \omega_{m}(\exp ) \\
{[\mathrm{eV}]}\end{array}$ & $\phi\left(x_{m}\right)$ & $\frac{I^{\prime}}{I}\left(x_{m}\right)$ \\
\hline 50 & 0.8 & 0.772 & 2.09 & 2.25 & 0.84 & 1.55 \\
\hline 40 & 1.6 & 0.951 & 2.58 & 2.48 & 3.00 & 1.9 \\
\hline 25 & 6.6 & 0.997 & 2.71 & 2.70 & 49.42 & 1.75 \\
\hline
\end{tabular}

(the best coincidence with the experimental data is attained at $\beta=28 \times 10^{-3} \frac{50^{2}}{(a[n m])^{2}}$ )

In Fig. 2, an estimation of normalized photo-current, $I^{\prime} / I$, with respect to the wave-length is presented for three sizes of metallic nanospheres ( $\mathrm{Au}$ ) deposited on a photo-active Si layer, with various structure parameters (the proximity parameter $\left.\beta=28 \times 10^{-3} \frac{50^{2}}{(a[n m])^{2}}\right):$

As indicated above, the relatively high value of $\frac{q_{m}}{q} \sim 10^{4} \frac{\beta 40}{H[n m]}$ enables a significant growth of the efficiency of the photo-energy transfer to the semiconductor, mediated by surface plasmons in nanoparticles deposited on the active layer, by increasing $\beta$ or reducing $H$ (at constant $n_{s}$ ). However, because of the fact that an enhancement of $\beta$ easily induces the overdamped regime, a greater perspective would be thus lowering $H$, the layer depth (cf. Fig.2 (left), where a significant growth of the photo-current with the lowering of the active layer depth $H$ illustrates the surface character of the effect). The overall behaviour of $I^{\prime} / I(\omega)=1+q_{m} / q$ calculated according to the relation (36), and depicted in the central panel in Fig. 2, agrees quite well with the experimental observations [7] (cf. inset in the central panel of Fig. 2), in the position, height and shape of the photo-current curves for distinct samples (the strongest enhancement is achieved for $a=40 \mathrm{~nm}$, as indicated in the central panel of Fig. 2), though $q_{m} / q$ is probably overestimated as the $q$ denominator would be greater for a doped real semiconductor structure but was not taken into account in the present calculus, similarly as surface effects; all of these would change the $q$ denominator as well as its energy dependence, especially for longer wavelengths, where the discrepancy between the theoretical model and the experimental data is noticeable.

When the metallic nanosphere would be immersed in the dielectric medium, the renormalisation of the resonance plasmon frequency takes place, which was analyzed widely for small clusters [11,34] and for larger particles, within the Mie-type approach $[22,23,24,35,36,37]$ or in RPA semiclassical method $[15,16]$.

It is evident that the PV efficiency enhancement due to energy transfer to semiconductor layer via plasmon excitations in metallic nanospheres is the surface-type effect, because of short range of near-field zone being essential to coupling of plasmons 
a)

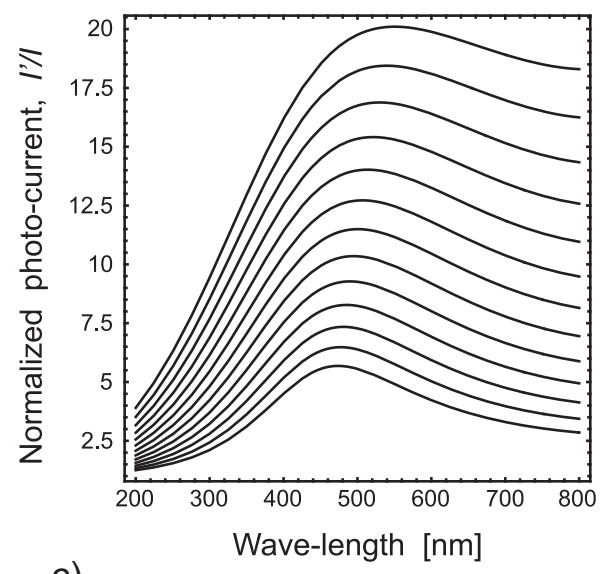

c)

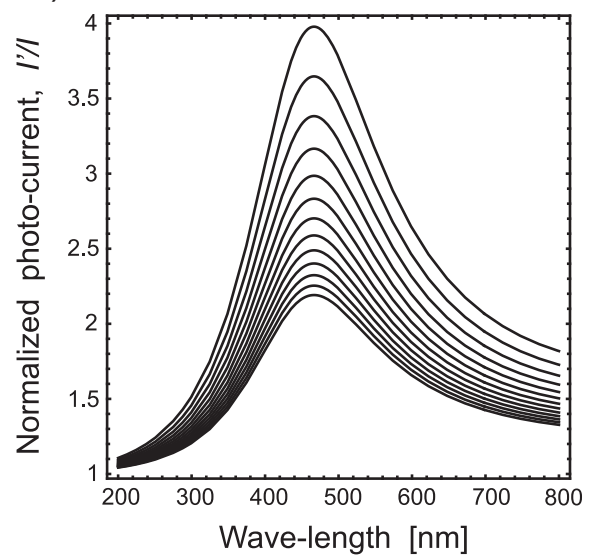

b)

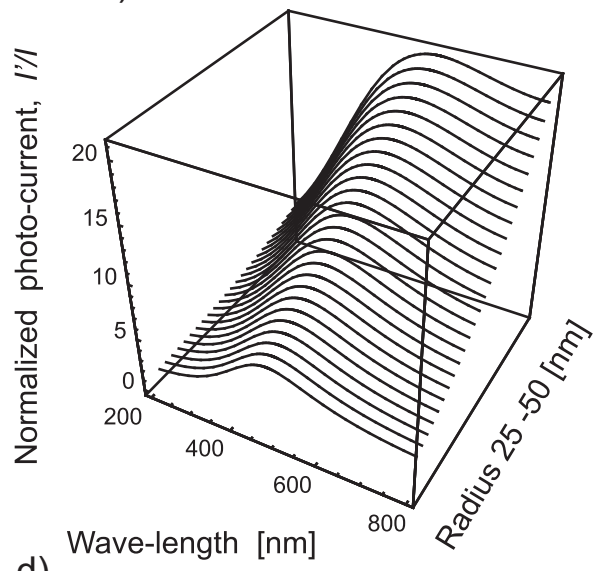

d)

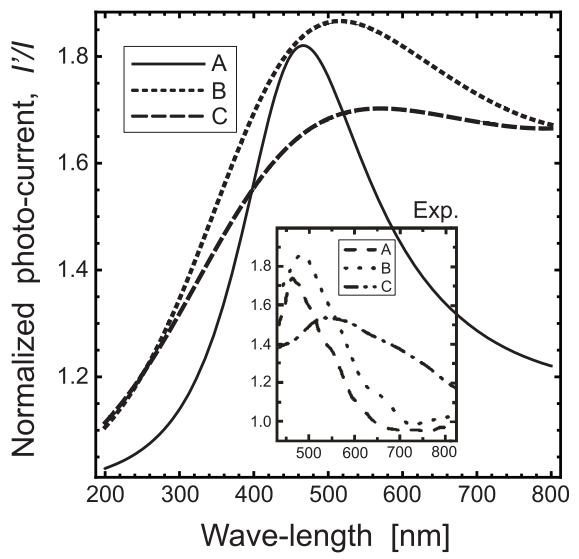

Figure 2. a) Dependence of the normalized photo-current $\frac{I^{\prime}}{I}(\lambda)$ with respect to the wave-length for growing radius $a$ from $25 \mathrm{~nm}$ (bottom) to $50 \mathrm{~nm}$ (upper), step $2 \mathrm{~nm}$ $\left(H=4 \mu \mathrm{m}, n_{s}=1.6 \times 10^{8} 1 / \mathrm{cm}^{2}\right)$ b) The same as in a) for better visualisation of the red-shift of the maximum with radius growth c) Dependence of the normalized photo-current $\frac{I^{\prime}}{I}(\lambda)$ with respect to the wave-length for growing layer depth $H$ from $4 \mu \mathrm{m}$ (upper) to $10 \mu \mathrm{m}$ (bottom), step $\left.0.5 \mu \mathrm{m}\left(a=20 \mathrm{~nm}, n_{s}=1.6 \times 10^{8} 1 / \mathrm{cm}^{2}\right) \mathrm{d}\right)$ Comparison with the experimental data-inset[7] for A: $a=25 \mathrm{~nm}$ (better fitting for $19 \mathrm{~nm}), n_{s}=6.6 \times 10^{8} 1 / \mathrm{cm}^{2}$, B: $a=40 \mathrm{~nm}, n_{s}=1.6 \times 10^{8} 1 / \mathrm{cm}^{2}$, C: $a=50 \mathrm{~nm}$, $n_{s}=0.8 \times 10^{8} 1 / \mathrm{cm}^{2}(H=3 \mu \mathrm{m})$

in surface-located nanocomponents with the substrate photo-active system. Therefore the plasmon effect would be of practical importance for a thin-film (e.g., III-V) solar cell technology. These systems are, however, relatively expensive and thus addressed to special applications rather, while for a commercial usage in the energy market, cheaper solutions are strongly desired. Of particular interest are 'plastic' solutions of polymer or dye chemical cells, which are, however, of low efficiency, as yet. An enhancement of their efficiency by only few percent would make these solutions competitive in an economical scale. Organic photovoltaics can be fabricated with an active polymer and a fullerene-based electron acceptor, arranged to separate charges dissociated by incident photons [38]. Though some analogy to semiconductor crystalline structure 
the significant differences in conjugated polymer based PV systems occur, resulting in lower efficiency of the latter ones. Smaller dielectric constant in polymers than in typical semiconductors causes that excitons are more strongly coupled and localized in organic materials. Transition of polymer molecular states (so-called HOMO-LUMO) [38] are the main source of carriers in conjugated polymers which are, however, of much lower mobility in comparison to ordinary semiconductor case. Thus the efficiency of plastic solar cell depends on many factors on all steps of the process, not only of the initial photon energy capturing [38]. Nevertheless, one can expect that application of plasmon metallic nanocomponents in polymer photo-active matrices would lead to similar efficiency enhancement to those in the case of inorganic semiconductors (the similar arguments behind the transition probability growth due to plasmon energy concentration in the near-field zone and contribution of indirect quantum jumps for non translational invariant near-field coupled nano-system hold). An instructive illustration of such a possibility would be recently reported control over optical transition efficiency in single molecule located closely to a nearby metallic nanosphere with surface plasmons [39]. The gain of efficiency was predicted up to a factor 5 but depending of the separation of molecule from the metallic nanoparticle (the best effect was for $20 \mathrm{~nm}$ separation for nanospheres of $40 \mathrm{~nm}$ radius) and geometry of both system polarizations. Despite of these constraints the effect indicates possibility of strengthening of PV effects by plasmon mediated energy transport also in other than semiconductor substrate situations. There is reported [40] increase of conjugated polymer cell efficiency from ca $1.7 \%$ to $2.5 \%$ by application of metallic (Ag) nano-coverings of photo-active surface, though competitive mechanism were manifested themselves via strong dependence of construction parameters.

\section{Undamped collective surface plasmon oscillations along metallic nanosphere chains}

Let us report here another effect connected with plasmon radiation in the near-field zone. One can consider plasmon excitations in a long linear chain of metallic nanospheres [21]. The transmission of a collective plasmon excitation of a wave-type along the chain can take place due to mutual coupling of nanospheres in the near-field zone. This results in hybrid state of plasmons with near-field electro-magnetic field called as plasmonpolariton $[2,21]$

For the model, let us consider a linear chain of metallic nanospheres with radii $a$ in a dielectric medium with dielectric constant $\varepsilon$. We assume that spheres are located along the $z$-axis direction equidistantly with the separation of the sphere centers $d>2 a$ $[21,41]$. At time $t=0$ we assume the excitation of plasmon oscillations via a Dirac delta, $\sim \delta(t)$, shaped signal of electric field. Taking into account the mutual interaction of the induced surface plasmons on the spheres via the radiation of dipole oscillations, we aim to determine the stationary state of the whole infinite chain. For a separation $d$ much shorter than the wavelength $\lambda$ of the e-m wave corresponding to surface plasmon 
self-frequency in a single nanosphere, the dipole type plasmon radiation can be treated within the near-field regime, at least for nearest neighbouring spheres. In the near-field region $a<R_{0}<\lambda$, the radiation of the dipole $\boldsymbol{D}(t)$ is not a planar wave (as it is for far-field region, $R_{0} \gg \lambda$ ) but is of only a retarded electric field (without a magnetic field) [27]:

$$
\boldsymbol{E}\left(\boldsymbol{R}, \boldsymbol{R}_{0}, t\right)=\frac{1}{\varepsilon R_{0}^{3}}\left[3 \boldsymbol{n}\left(\boldsymbol{n} \cdot \boldsymbol{D}\left(\boldsymbol{R}, t-\frac{R_{0}}{v}\right)\right)-\boldsymbol{D}\left(\boldsymbol{R}, t-\frac{R_{0}}{v}\right)\right],
$$

$\boldsymbol{R}$ is the position of the sphere (center) irradiating e-m energy due to its dipole surface plasmon oscillations, $\boldsymbol{R}_{0}$ is the position of another sphere (center), with respect to the center of the former one, where the field $\boldsymbol{E}\left(\boldsymbol{R}, \boldsymbol{R}_{0}, t\right)$ is given by the above formula, $R_{0}<\lambda, \boldsymbol{n}=\boldsymbol{R}_{0} / R_{0}, v=c / \sqrt{\varepsilon}=c / n_{0}$.

When both vectors $\boldsymbol{R}$ and $\boldsymbol{R}_{0}$ are along the $z$-axis (the linear chain) the above equation can be resolved as:

$$
E_{\alpha}\left(\boldsymbol{R}, \boldsymbol{R}_{0}, t\right)=\frac{\sigma_{\alpha}}{\varepsilon R_{0}^{3}} D_{\alpha}\left(\boldsymbol{R}, t-\frac{R_{0}}{v}\right),
$$

where $\alpha=(x, y, z), \sigma_{x}=\sigma_{y}=-1$ and $\sigma_{z}=2$. Assuming that the $z$-axis origin coincides with the center of one sphere in the chain, for the $l^{\text {th }}$ sphere located in the point $\boldsymbol{R}_{l}=(0,0, l d)$, an electric field caused by neighbouring spheres, $\boldsymbol{E}\left(\boldsymbol{R}_{m}, \boldsymbol{R}_{m l}, t\right)$, and the Lorentz friction force caused by self-radiation, $\boldsymbol{E}_{L}\left(\boldsymbol{R}_{l}, t\right)$, have to be accounted for. By virtue of Eq. (23) the equation for the surface plasmon oscillation of the $l^{\text {th }}$ sphere is

$$
\begin{aligned}
& {\left[\frac{\partial^{2}}{\partial t^{2}}+\frac{2}{\tau_{0}} \frac{\partial}{\partial t}+\omega_{1}^{2}\right] \boldsymbol{q}\left(\boldsymbol{R}_{l}, t\right)} \\
& =\frac{e n_{e}}{m} \sum_{m=-\infty}^{m=\infty} \sum_{m \neq l, R_{m l}<\lambda} \boldsymbol{E}\left(\boldsymbol{R}_{m}, \boldsymbol{R}_{m l}, t\right)+\frac{e n_{e}}{m} \boldsymbol{E}_{L}\left(\boldsymbol{R}_{l}, t\right),
\end{aligned}
$$

provided that the dipole field of the $m^{\text {th }}$ sphere can be treated as homogeneous over the $l^{\text {th }}$ sphere and the sum over $m$ is confined by the distance of the $m^{\text {th }}$ sphere from the $l^{\text {th }}$ sphere not exceeding the near-field range $(\sim \lambda)$. In the case of the equidistant chain, $R_{l}=l d$ and $R_{m l}=|l-m| d$, and using Eqs (38), (22) and (26), one can rewrite Eq. (39) in the form:

$$
\begin{aligned}
& {\left[\frac{\partial^{2}}{\partial t^{2}}+\frac{2}{\tau_{0}} \frac{\partial}{\partial t}-\frac{2}{3 \omega_{1}}\left(\frac{\omega_{1} a}{v}\right)^{3} \frac{\partial^{3}}{\partial t^{3}}+\omega_{1}^{2}\right] q_{\alpha}(l d, t)} \\
& =\sigma_{\alpha} \omega_{1}^{2} \frac{a^{3}}{d^{3}} \sum_{m=-\infty, m \neq l,|l-m| d<\lambda}^{m=\infty} \frac{q_{\alpha}\left(m d, t-\frac{d}{v}|l-m|\right)}{|l-m|^{3}},
\end{aligned}
$$

here $\alpha=x, y$, which describe the transversal plasmon modes and $\alpha=z$, which describes the longitudinal mode; $\omega_{1}^{2}=\frac{\omega_{p}^{2}}{3 \varepsilon}=\frac{4 \pi n_{e} e^{2}}{3 \varepsilon m}, v=\frac{c}{\sqrt{\varepsilon}}$. The above equation coincides with the similar considered by Atwater [21,41], if one assumes that $\frac{4 \pi}{3} a^{3} n_{e}=N=1$ and neglects the retardation of the field.

Taking into account the periodicity of the infinite chain, one can consider the solution of the above equation in the form

$$
q_{\alpha}(l d, t)=\tilde{q}_{\alpha}(k, t) e^{-i k l d} .
$$


The right-hand-side term in Eq. (40) attains the form

$$
\begin{aligned}
& \sum_{m=-\infty, m \neq l}^{\infty} \frac{q_{\alpha}\left(m d, t-\frac{d}{v}|l-m|\right)}{|l-m|^{3}}=\sum_{m=-\infty}^{l-1} \frac{q_{\alpha}\left(m d, t-\frac{d}{v}|l-m|\right)}{|l-m|^{3}}+\sum_{m=l+1}^{\infty} \frac{q_{\alpha}\left(m d, t-\frac{d}{v}|l-m|\right)}{|l-m|^{3}} \\
& =2 e^{-i k l d} \sum_{m=1}^{\infty} \frac{\cos (m k d)}{m^{3}} \tilde{q}_{\alpha}(k, t-m d / v) .
\end{aligned}
$$

Thus the Eq. (40) can be written as follows:

$$
\begin{aligned}
& {\left[\frac{\partial^{2}}{\partial t^{2}}+\frac{2}{\tau_{0}} \frac{\partial}{\partial t}-\frac{2}{3 \omega_{1}}\left(\frac{\omega_{1} a}{v}\right)^{3} \frac{\partial^{3}}{\partial t^{3}}+\omega_{1}^{2}\right] \tilde{q}_{\alpha}(k, t)} \\
& =\sigma_{\alpha} \omega_{1}^{2} \frac{a^{3}}{d^{3}} 2 \sum_{m=1, m d<\lambda}^{\infty} \frac{\cos (m k d)}{m^{3}} \tilde{q}_{\alpha}(k, t-m d / v) .
\end{aligned}
$$

This equation is linear and therefore we look for the solutions of the shape: $\tilde{q}_{\alpha}(k, t)=\tilde{Q}_{\alpha}(k) e^{i \omega_{\alpha} t}$, and we arrive at the condition,

$$
-\omega_{\alpha}^{2}+\frac{2 i \omega_{\alpha}}{\tau_{\alpha}\left(\omega_{\alpha}\right)}+\tilde{\omega}_{\alpha}^{2}\left(\omega_{\alpha}\right)=0
$$

where

$$
\tilde{\omega}_{\alpha}^{2}\left(\omega_{\alpha}\right)=\omega_{1}^{2}\left[1-\frac{2 \sigma_{\alpha} a^{3}}{d^{3}} \sum_{m=1, m d<\lambda}^{\infty} \frac{\cos (m k d)}{m^{3}} \cos \left(\frac{\omega_{\alpha} m d}{v}\right)\right]
$$

and

$$
\frac{1}{\tau_{\alpha}\left(\omega_{\alpha}\right)}=\frac{1}{\tau_{0}}+\frac{\omega_{1}^{2} a}{3 v}\left(\frac{\omega_{\alpha} a}{v}\right)^{2}+\sigma_{\alpha} \omega_{1}^{2} \frac{a^{3}}{d^{3}} \sum_{m=1, m d<\lambda}^{\infty} \frac{\cos (m k d)}{m^{3}} \frac{\sin \left(\frac{\omega_{\alpha} m d}{v}\right)}{\omega_{\alpha}} .
$$

If we confine the sum in Eq. (44) to $m=1$ (the nearest neighbour approximation) we get

$$
\tilde{\omega}_{\alpha}^{2}\left(\omega_{\alpha}\right) \simeq \omega_{1}^{2}\left[1-\frac{2 \sigma_{\alpha} a^{3}}{d^{3}} \cos (k d) \cos \left(\frac{\omega_{\alpha} d}{v}\right)\right]
$$

and from Eq. (45),

$$
\frac{1}{\tau_{\alpha}\left(\omega_{\alpha}\right)}=\frac{1}{\tau_{0}}+\frac{\omega_{1}^{2} a^{3}}{4 v d^{2}}\left[\left(\frac{\omega_{\alpha} d}{v}\right)^{2}-(k d-\pi)^{2}+\frac{\pi^{2}}{3}\right], \text { for } \alpha=x, y
$$

and

$$
\frac{1}{\tau_{z}\left(\omega_{z}\right)}=\frac{1}{\tau_{0}}+\frac{\omega_{1}^{2} a^{3}}{2 v d^{2}}\left[\left(\frac{\omega_{z} d}{v}\right)^{2}+(k d-\pi)^{2}-\frac{\pi^{2}}{3}\right], \text { for } \alpha=z .
$$

In the derivation of the two above formulae the following summation was performed $[42]$ :

$$
\begin{aligned}
& \frac{1}{\omega_{\alpha}} \sum_{m=1}^{\infty} \frac{\cos (m k d)}{m^{3}} \sin \left(\frac{\omega_{\alpha} m d}{v}\right)=\frac{1}{2 \omega_{\alpha}} \sum_{m=1}^{\infty} \frac{1}{m^{3}}\left[\sin \left(k m d+\omega_{\alpha} m d / v\right)-\sin \left(k m d-\omega_{\alpha} m d / v\right)\right] \\
& =\frac{d}{v}\left[\frac{\pi^{2}}{6}-\frac{\pi}{2} k d+\frac{k^{2} d^{2}}{4}+\frac{\omega_{\alpha}^{2} d^{2}}{2 v^{2}}\right] .
\end{aligned}
$$

Because the terms in the sum drop quickly to zero, the above formula approximates well the sum with limitation $m d<\lambda$. 
Assuming now $\omega_{\alpha}=\omega_{\alpha}^{\prime}+i \omega_{\alpha}^{\prime \prime}$, the Eq. (43) gives the dependence of $\omega_{\alpha}^{\prime}$ and $\omega_{\alpha}^{\prime \prime}$ on $k$. The general solution of Eq. (40) attains the form,

$$
q_{\alpha}(l d, t)=\sum_{n=1}^{N_{s}} \tilde{Q}_{\alpha}\left(k_{n}\right) e^{i\left(\omega_{\alpha}^{\prime}\left(k_{n}\right) t-k_{n} l d\right)-\omega_{\alpha}^{\prime \prime}\left(k_{n}\right) t},
$$

where $k_{n}=\frac{2 \pi n}{N_{s} d}, L=N_{s} d$ is the assumed length of the chain with $N_{s}$ spheres, when periodic (Born-Karman type) boundary conditions are imposed. The components of Eq. (49) describe monochromatic waves with wavelength $\lambda_{n}=\frac{2 \pi}{k_{n}}=\frac{L}{n}$, which are analogous to planar waves in crystals, when damping is not big, i.e., when $\omega_{\alpha}^{\prime \prime} \ll \omega_{\alpha}^{\prime}$. Provided with this inequality, one can approximate:

for the transversal modes $(\alpha=x, y)$

$$
\begin{aligned}
& \left(\omega_{\alpha}^{\prime}\right)^{2}=\tilde{\omega}_{\alpha}^{2}=\omega_{1}^{2}\left[1+\frac{2 a^{3}}{d^{3}} \cos (k d) \cos \left(\omega_{\alpha}^{\prime} d / v\right)\right], \\
& \omega_{\alpha}^{\prime \prime}=\frac{1}{\tau_{\alpha}}=\frac{1}{\tau_{0}}+\frac{\omega_{1}^{2} a^{3}}{4 v d^{2}}\left[\left(\frac{\omega_{\alpha}^{\prime} d}{v}\right)^{2}-(k d-\pi)^{2}+\frac{\pi^{2}}{3}\right],
\end{aligned}
$$

and for the longitudinal mode $(\alpha=z)$

$$
\begin{aligned}
& \left(\omega_{z}^{\prime}\right)^{2}=\tilde{\omega}_{z}^{2}=\omega_{1}^{2}\left[1-\frac{4 a^{3}}{d^{3}} \cos (k d) \cos \left(\omega_{z}^{\prime} d / v\right)\right], \\
& \omega_{z}^{\prime \prime}=\frac{1}{\tau_{z}}=\frac{1}{\tau_{0}}+\frac{\omega_{1}^{2} a^{3}}{2 v d^{2}}\left[\left(\frac{\omega_{\alpha}^{\prime} d}{v}\right)^{2}+(k d-\pi)^{2}-\frac{\pi^{2}}{3}\right] .
\end{aligned}
$$

From Eqs (51) and (53) it follows that $\omega_{\alpha}^{\prime \prime}$ can change its sign. In the case of $\omega_{\alpha}^{\prime \prime}<0$ the oscillations are destabilized, which could be avoided by inclusions of some nonlinear terms neglected in the expression for the Lorentz friction, which in more accurate form [27] includes also a small nonlinear term with respect to $D$, aside from the term with $\frac{\partial^{3} D}{\partial t^{3}}$. Including this will result in damping of too highly rising oscillations and will lead to stable oscillation amplitude. Due to this stabilization caused by nonlinear effects, undamped wave modes of dipole oscillations will propagate in the chain in the region of parameters where $\omega_{\alpha}^{\prime \prime} \leq 0$ (and with fixed amplitude accommodated by the nonlinear term). The condition $\omega_{\alpha}^{\prime \prime}=\frac{1}{\tau_{\alpha}}=0$, for critical parameters, resolves into:

$$
\left(\frac{\omega_{\alpha} d}{v}\right)^{2}=(k d-\pi)^{2}-\frac{\pi^{2}}{3}-\frac{4 v d^{2}}{\tau_{0} \omega_{1}^{2} a^{3}},
$$

for $\alpha=(x, y)$ and for $\alpha=z$,

$$
\left(\frac{\omega_{z} d}{v}\right)^{2}=-(k d-\pi)^{2}+\frac{\pi^{2}}{3}-\frac{2 v d^{2}}{\tau_{0} \omega_{1}^{2} a^{3}} .
$$

$\left(\frac{\omega_{\alpha} d}{v}\right)$ obtained from the above equations leads to determination of the dependence of wave vector $k$ with respect to parameters $d$ and $a$, via Eqs (50)-(53). Solutions for these equations, found numerically for the chain of Ag nanospheres, are depicted in Fig. 3. 
$\mathrm{Ag}, \mathrm{d} / \mathrm{a}=3$
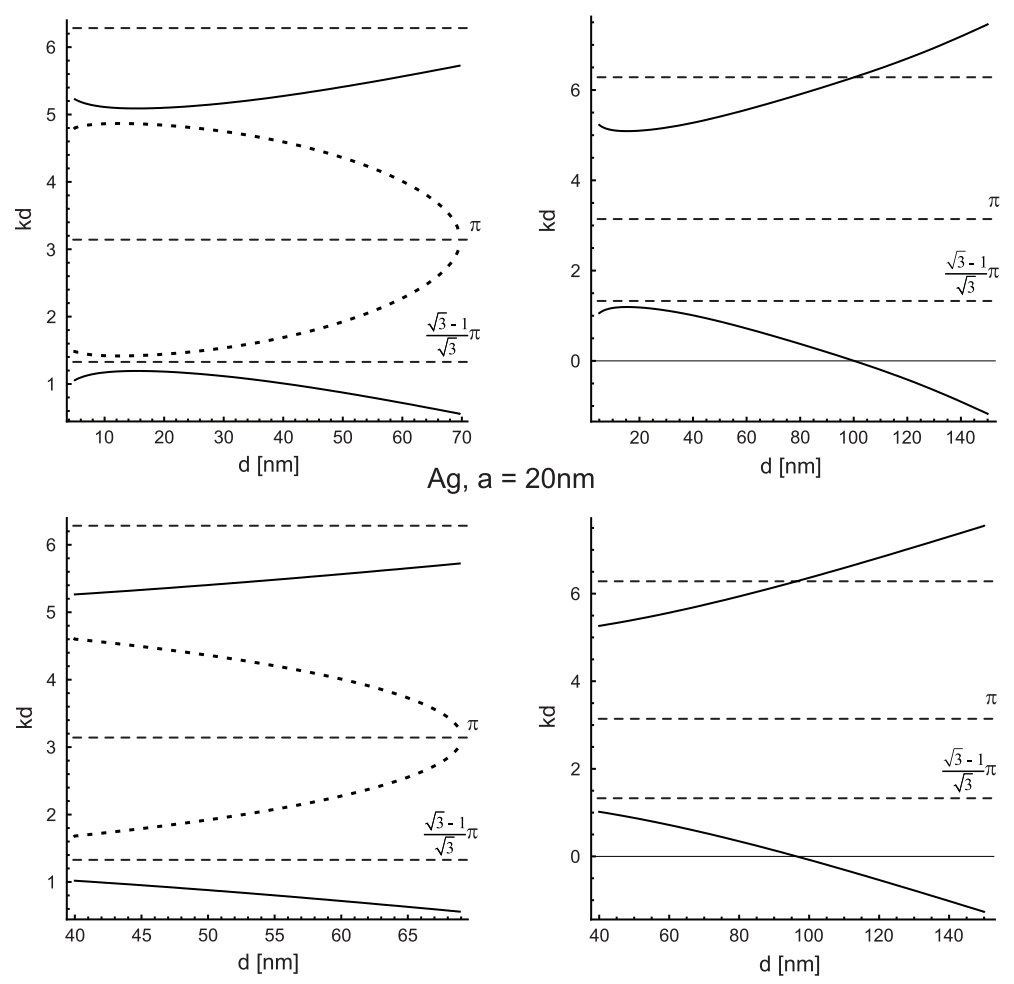

Figure 3. Wave vector $k d$ for undamped surface plasmon propagation along the metallic nano-sphere chain versus sphere separation $d$ at (top) constant $d / a=3$, with $a$ the sphere radius, and (bottom) for $a=20 \mathrm{~nm}$ for $\mathrm{Ag}$ sphere chains, respectively; transversal modes are shown by solid lines, while longitudinal modes are shown by dotted lines

Undamped plasmon waves in the chain appear if $d<d_{\text {max }}$ and have $k=\pi / d$, $d_{\text {max }}=98.5(68.8) \mathrm{nm}$ for transversal(longitudinal) modes. For example, for Ag spheres with the radius $a=20 \mathrm{~nm}$ and the separation $d=60 \mathrm{~nm}$, undamped transversal modes appear for $0 \leq k d \leq \pi / 4$ or $3 \pi / 4 \leq k d \leq 2 \pi$ and an undamped longitudinal mode for $3 \pi / 4 \leq k d \leq 5 \pi / 4$.

Let us underline that the determined undamped plasmon oscillation wave modes would explain similar, numerically observed behaviour [25]. Within that numerical analysis two types of collective surface plasmons with distinct propagation along the nano-sphere chain were identified, and called as quasistatic (ordinary) and nonquasistatic (extraordinary) surface plasmon modes. The quasistatic modes were damped while nonquasistatic ones were undamped (and additionally, the latter turn out to be relatively robust against the disorder in the chain) [25]. One can associate these observations with undamped collective plasmon modes with the negative imaginary part of the frequency, accessible in the described above regions of the chain parameters and the wave vector values (stabilized by nonlinear terms of Lorentz friction at a certain amplitude), which are, however, accompanied by ordinary damped modes with the positive imaginary frequency part. 


\section{Conclusions}

We have analyzed various channels of surface plasmon damping. Inclusion of irradiation losses due to Lorentz friction of oscillating electrons gives the satisfactory explanation of the scale and radius dependence of plasmon resonance in metallic spheres with the radii $>10 \mathrm{~nm}$. The most effective channel for the surface plasmon damping turns out to be the dipole-type near-field coupling of the surface dipole plasmons with semiconductor substrate, on which metallic nanospheres would be deposited, e.g., in nanomodified diode-type systems. Due to the nano-scale of the spheres for this coupling the momentum is not conserved, which results in a strong enhancement of the interband transition probability (because all indirect electron transitions between the valence and conductivity bands in the substrate semiconductor have to be accounted for, provided energy conservation alone). This agrees with the experimental data referring to a significant growth of the energy transfer from surface plasmons in metallic nanoparticles to the semiconductor substrate. In the presented model nanosphere surface plasmons couple with substrate charges (band electrons in a substrate semiconductor) via photonless short range e-m dipole interaction with very quick timing (thus very effective) - as confirmed by the time-resolved spectroscopy measurements. The strong enhancement of the efficiency results from the nanoscale-induced incommensurability, leading to all momentum-indirect interband transitions, not allowed for the interaction of band electrons with the original incident planar wave photons as in an ordinary photoeffect. The type of dipole coupling is connected here with a specific e-m field gauge in the vicinity of the nanosphere within the distance lower than the wave-length (thus 'inside' the single photon), crucially distinct than for the planar wave (in the latter case, only the vector potential can be used, which is impossible in the former case). The above schematically described scenario qualitatively fits with the experimentally observed behaviour and elucidates the timing of the particular steps of the energytransfer-processes, including the mediating role of metallic nanosphere surface plasmons. The relevant time rates can be estimated within the standard quantum mechanical attitude of the Fermi-golden-rule-type.

The another effect connected with near-field coupling is a plasmon-polariton collective excitation in metal-dielectric interfaces, including periodic nano-particle systems, with convenient for applications sub-diffraction wave length (due to lower group velocity in comparison to light velocity) We have considered also such a wave-type collective plasmon oscillations in the metallic nano-sphere chain. The undamped region of wave propagation through the chain is found for a certain sphere separation in the chain with the corresponding wavelength of plasmon waves. This phenomenon confirms similar behaviour observed by numerical simulations.

The novelty of presented results consists in an analysis of the energy transfer of plasmon energy from nanosphere to substrate semiconductor band electrons, assessed in the framework of Fermi golden rule, and resulting in a different transition probability than for ordinary photo-effect. This agrees with the experimental observations of photo- 
current enhancement in the Si diode setup with active surface covered by sparsely distributed metallic nanospheres with radius of several tens $\mathrm{nm}$, both with respect to maximum of photo-current enhancement and the overall dispersion shape. The other effect closely related with the near-field zone coupling of the surface plasmons in metallic nanospheres is energy transfer along a chain of metallic nanocomponents. Described in the paper, an identification of an undamped collective mode of surface plasmons nearfield coupled in the chain of metallic nanospheres would be of some significance for subdiffraction arrangement of electro-photonic circuits and possibly for energy transport in novel constructions of solar cells, especially for plastic cells where low mobility and short free path of carriers are severe restrictions imposed on their efficiency.

[1] Barnes W L, Dereux A and Ebbesen T W 2003 Nature 424824

[2] Zayats A V, Smolyaninov I I and Maradudin A A 2005 Phys. Rep. 408131

[3] Maier S A Plasmonics: Fundamentals and Applications (Springer, Berlin, 2007)

[4] Pillai S, Catchpole K R, Trupke T, Zhang G, Zhao J and Green M A 2006 Appl. Phys. Lett. 88 161102

[5] Westphalen M, Kreibig U, Rostalski J, Lüth H and Meissner D 2003 Sol. Energy Mater. Sol. Cells 61 97; Gratzel M 2003 J. Photochem. Photobiol. C: Photochem. Rev. 4145

[6] Stuart H R and Hall D G 1998 Appl. Phys. Lett. 73 3815; Stuart H R and Hall D G 1998 Phys. Rev. Lett. 80 5663; Stuart H R and Hall D G 1996 Appl. Phys. Lett. 692327

[7] Schaadt D M, Feng B and Yu E T 2005 Appl. Phys. Lett. 86063106

[8] Okamoto K, Niki I, Shvartser A, Narukawa Y, Mukai T and Scherer A 2004 Nature Mat. 3 601; Okamoto K, Niki I, Scherer A, Narukawa Y, Mukai T and Kawakami Y 2005 Appl. Phys. Lett. 87071102

[9] Wen C, Ishikawa K, Kishima M and Yamada K 2000 Sol. Cells 61339

[10] Mie G 1908 Ann. Phys. 25377

[11] Brack M 1993 Rev. of Mod. Phys. 65677

[12] Ekardt W 1985 Phys. Rev B $\mathbf{3 1} 6360$

[13] Brack M 1989 Phys. Rev. B 393533

[14] Kresin V V 1992 Phys. Rep. 2201

[15] Jacak J, Krasnyj J, Jacak W, Gonczarek R, Chepok A and Jacak L 2010 Phys. Rev. B 82035418

[16] Jacak W, Krasnyj J, Jacak J, Gonczarek R, Chepok A, Jacak L, Hu D and Schaadt D $2010 \mathrm{~J}$. Appl. Phys. 107124317

[17] Jacak L, Krasnyj J and Chepok A 2009 Fiz. Nisk. Temp. 35491

[18] Yannouleas C, Broglia R A, Brack M and Bortignon P F 1989 Phys. Rev. Lett. 63 255; Weick G, Ingold G L, Jalabert R A and Weinmann D 2006 Phys. Rev. B 74165421

[19] Pines D Elementary Excitations in Solids (ABP Perseus Books, Massachusetts, 1999)

[20] Pines D and Bohm D 1952 Phys. Rev. 85 338; Pines D and Bohm D 1952 Phys. Rev. 92609

[21] Brongersma M L, Hartman J W and Atwater H A 2000 Phys. Rev. B 62 R16356

[22] Bohren C F, Huffman D R Absorption and Scattering of Light by Small Particles (Wiley, New York, 1983)

[23] Petrov J I Physics of Small Particles (Nauka, Moscow, 1984)

[24] Pitarke J M, Silkin V M, Chulkov E V and Echenique P M 2007 Rep. Prog. Phys. 701

[25] Markel V A and Sarychev A K 2007 Phys. Rev. B 75085426

[26] Ekardt W 1984 Phys. Rev. Lett. 521925

[27] Landau L D and Lifshitz E M Field Theory (Nauka, Moscow, 1973)

[28] Ekardt W 1986 Phys. Rev. B 338803

[29] Garcia de Abajo F J 2010 Rev. Mod. Phys. 82209

[30] Garcia de Abajo F J 2008 J. Phys. Chem. C 11217983

[31] Kiriejew P S Physics of Semiconductors (PWN, Warsaw, 1969) 
[32] Sundararajan S P, Grandy N K, Mirin N and Halas N J 2008 Nano Lett. 8624

[33] Losurdo M, Giangregorio M M, Bianco G V, Sacchetti A, Capezzuto P and Bruno G 2009 Solar Energy Mat. 83 Solar Cells 931749

[34] Rubio A and Serra L 1993 Phys. Rev. B 4818222

[35] Noguez C 2007 J. Phys. Chem C 1113806

[36] Noguez C 2005 Opt. Mat. 271204

[37] Kelly K L, Coronado E, Zhao L L and Schatz G 2003 J. Phys. Chem C 107668

[38] Yoon W J, Studies of Conjugated Polymer Semiconductor Electronics and Optoelectronics (PhD Dissertation, Ohio State Univ, 2009); Mayer A, Scully S, Hardin B, Rowell M, and McGehee M 2007 Materials Today 1028

[39] Anger P, Bharadwaj P and Novotny L 2006 Phys. Rev. Lett. 96113002

[40] Morfa A J, Rowlen K L, Reilly T H, Romero M J and Lagemaat J 2008 Appl. Phys. Lett. 92 013504

[41] Maier S A, Kik P G and Atwater H A 2003 Phys. Rev. B 67205402

[42] Gradstein I S and Rizik I M Tables of Integrals (Fizmatizdat, Moscow, 1962) 